ANNALES

POLONICI MATHEMATICI

$82.3(2003)$

\title{
New versions of curvature and torsion formulas for the complete lifting of a linear connection to Weil bundles
}

\author{
by A. Ntyam and J. Wouafo Kamga (Yaoundé)
}

Abstract. New versions of Slovák's formulas expressing the covariant derivative and curvature of the linear connection $\mathcal{T}_{A} \Gamma$ are presented.

1. Introduction. Let $T_{A}$ be a Weil functor and consider a linear connection $\Gamma$ on a vector bundle $(E, M, \pi)$; one defines (see [3] or [7]) the linear connection $\mathcal{T}_{A} \Gamma$ on $\left(T_{A} E, T_{A} M, T_{A} \pi\right)$ by

$$
\mathcal{T}_{A} \Gamma=\kappa_{E} \circ T_{A} \Gamma \circ\left(\kappa_{M}^{-1} \times_{T_{A} M} \operatorname{id}_{T_{A} E}\right): T T_{A} M \times_{T_{A} M} T_{A} E \rightarrow T T_{A} E,
$$

where $\kappa: T_{A} T \rightarrow T T_{A}$ is the canonical flow natural equivalence.

The main results of this paper are Propositions 6 and 7 giving new versions of formulas expressing the covariant derivative and curvature of $\mathcal{T}_{A} \Gamma$. In the case $E=T M$, we obtain some results of [2] and [1] (Corollaries 3 and 4$)$.

\section{Weil functor}

\subsection{Weil algebra}

Definition 1. A Weil algebra is a finite-dimensional quotient of the algebra of germs $\mathcal{E}_{p}=C_{0}^{\infty}\left(\mathbb{R}^{p}, \mathbb{R}\right)\left(p \in \mathbb{N}^{*}\right)$.

We denote by $\mathcal{M}_{p}$ the maximal ideal of $\mathcal{E}_{p}$.

EXAmPLE 1. (1) $\mathbb{R}$ is a Weil algebra since it is canonically isomorphic to the quotient $\mathcal{E}_{p} / \mathcal{M}_{p}$.

(2) $J_{0}^{r}\left(\mathbb{R}^{p}, \mathbb{R}\right)=\mathcal{E}_{p} / \mathcal{M}_{p}^{r+1}$ is a Weil algebra.

2.2. Covariant description of a Weil functor $T_{A}: \mathcal{M} f \rightarrow \mathcal{F M}$. We write $\mathcal{M} f$ for the category of differentiable manifolds and mappings of class $C^{\infty}$;

2000 Mathematics Subject Classification: 58A05, 58A32.

Key words and phrases: Weil bundle, tensor field, linear connection, prolongations. 
furthermore, $\mathcal{F M}$ is the category of fibered manifolds and fibered manifold morphisms.

Let $A=\mathcal{E}_{p} / I$ be a Weil algebra and consider a manifold $M . \operatorname{In} C^{\infty}\left(\mathbb{R}^{p}, M\right)$ one defines an equivalence relation $\mathcal{R}$ by: $\varphi \mathcal{R} \psi$ if and only if $\varphi(0)=\psi(0)=x$ and for any $[h]_{x} \in C_{x}^{\infty}(M, \mathbb{R}),[h]_{x} \circ[\psi]_{0}-[h]_{x} \circ[\varphi]_{0} \in I$.

The equivalence class of $\varphi$ is denoted by $j_{A} \varphi$ and is called the A-velocity at 0 of $\varphi$; the class $j_{A} \varphi$ depends only on the germ of $\varphi$ at 0 . The quotient $C^{\infty}\left(\mathbb{R}^{p}, M\right) / \mathcal{R}$ is denoted by $T_{A} M$.

The mapping $\pi_{A, M}: T_{A} M \rightarrow M, j_{A} \varphi \mapsto \varphi(0)$, defines a bundle structure on $T_{A} M$ and for any differentiable mapping $f: M \rightarrow N$, one defines a bundle morphism $T_{A} f: T_{A} M \rightarrow T_{A} N$ (over $f$ ) by $T_{A} f\left(j_{A}(\varphi)\right)=j_{A}(f \circ \varphi)$.

The correspondence $T_{A}: \mathcal{M} f \rightarrow \mathcal{F} \mathcal{M}$ is a product preserving bundle functor (see [3]).

ExAmple 2. If $A=J_{0}^{r}\left(\mathbb{R}^{p}, \mathbb{R}\right)$, then $T_{A}$ is equivalent to the functor $T_{p}^{r}$ of $(p, r)$-velocities, and if $A=\mathcal{E}_{p} / \mathcal{M}_{p}^{2}$, then $T_{A}=T$, the tangent bundle functor.

2.3. The canonical flow-natural equivalence. Let $T_{A}, T_{B}$ be two Weil functors. Our purpose here is to make explicit a natural equivalence

$$
\kappa: T_{A} \circ T_{B} \rightarrow T_{B} \circ T_{A} .
$$

Lemma 1 ([3]). Let $M$ be a manifold. For any $\zeta=j_{A} \varphi \in T_{A} T_{B} M$, there is a differentiable mapping $\Phi: \mathbb{R}^{p} \times \mathbb{R}^{q} \rightarrow M$ such that $\varphi(z)=j_{B} \Phi_{z}$ in a neighbourhood of $0 \in \mathbb{R}^{p}$.

By this lemma, one defines $\kappa: T_{A} \circ T_{B} \rightarrow T_{B} \circ T_{A}$ as follows:

$$
\kappa_{M}(\zeta)=j_{B} \eta
$$

where $\eta: \mathbb{R}^{q} \rightarrow T_{A} M, t \mapsto j_{A} \Phi^{t}$. It is a well-defined natural equivalence. In particular, for $T_{B}=T$, we obtain the canonical flow-natural equivalence.

3. Prolongations of tensor fields of type $(1, s)$. In this section, $A$ is a Weil algebra, i.e. $\mathcal{E}_{p} / I$ with $\mathcal{M}_{p} \supset I \supset \mathcal{M}_{p}^{r+1}$ and $r$ minimal; $\mathcal{V B}$ is the category of vector bundles and vector bundle homomorphisms. The module of differentiable sections of a vector bundle $(E, M, \pi)$ is denoted here by $\operatorname{Sec}(M, E)$.

3.1. The functor $T_{A}: \mathcal{V B} \rightarrow \mathcal{V B}$. It is defined as follows:

$$
T_{A}(E, M, \pi)=\left(T_{A} E, T_{A} M, T_{A} \pi\right), \quad T_{A}(\bar{f}, f)=\left(T_{A} \bar{f}, T_{A} f\right)
$$

see $([2]$ and $[4])$.

3.2. Natural transformations $\chi_{\alpha}: T_{A} \rightarrow T_{A}$. Consider a vector bundle $(E, M, \pi)$. For any multi-index $\alpha \in \mathbb{N}^{p}$ such that $|\alpha| \leq r$, we put

$$
\left(\chi_{\alpha}\right)_{E}\left(j_{A} f\right)=j_{A}\left(z^{\alpha} f\right)
$$


where $f: \mathbb{R}^{p} \rightarrow E$ is $C^{\infty}$ and $z^{\alpha} f: \mathbb{R}^{p} \rightarrow E, z \mapsto z^{\alpha} f(z) \in E_{\pi(f(0))}$. One defines in this way some natural transformations $\chi_{\alpha}: T_{A} \rightarrow T_{A}$, since each $\left(\chi_{\alpha}\right)_{E}$ is a vector bundle morphism over $\operatorname{id}_{T_{A} M}$.

Proposition 1. For any multi-index $\alpha \in \mathbb{N}^{p}$ such that $|\alpha| \leq r$, the diagram

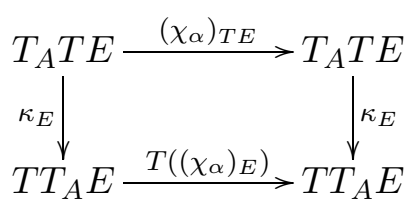

is commutative, where $\kappa: T_{A} T \rightarrow T T_{A}$ is the canonical flow-natural equivalence.

3.3. Prolongation of tensor fields of type $(1,0)$. Consider a $\mathcal{V B}$-object $(E, M, \pi)$ and a differentiable section $S: M \rightarrow E$. One defines the following prolongations of $S$ on $\left(T_{A} E, T_{A} M, T_{A} \pi\right)$ :

$$
S^{(0)}=T_{A} S\left(\text { since }\left(\chi_{0}\right)_{E}=\operatorname{id}_{T_{A} E}\right), \quad S^{(\alpha)}=\left(\chi_{\alpha}\right)_{E} \circ T_{A} S, 1 \leq|\alpha| \leq r,
$$

where $\chi_{\alpha}$ is the natural transformation (2). If $|\alpha|>r$, then $S^{(\alpha)}:=0_{T_{A} E}$.

Let $\varphi: \pi^{-1}(U) \rightarrow U \times \mathbb{R}^{n}$ be a local trivialisation of $E$ and $\varepsilon_{j}(x)=$ $\varphi^{-1}\left(x, e_{j}\right), 1 \leq j \leq n$, a basis of sections of $E$ over $U$ associated to $\varphi$ (here $\left(e_{j}\right), 1 \leq j \leq n$, is the usual basis of $\mathbb{R}^{n}$ ). Using the identification $T_{A}\left(U \times \mathbb{R}^{n}\right) \cong T_{A} U \times T_{A} \mathbb{R}^{n}$, one defines a family of sections

$$
\left(\varepsilon_{j, \alpha}\right), \quad|\alpha| \leq r, 1 \leq j \leq n,
$$

of $T_{A} E$ over $T_{A} U$ by

$$
\varepsilon_{j, \alpha}(\widetilde{x})=T_{A} \varphi^{-1}\left(\widetilde{x}, e_{j \alpha}\right),
$$

where $e_{j \alpha}=j_{A}\left(z^{\alpha} e_{j}\right)$. Then

$$
\varepsilon_{j}^{(\alpha)}=\varepsilon_{j, \alpha}, \quad 1 \leq j \leq n \text { and }|\alpha| \leq r,
$$

and we deduce

Proposition 2. The $S^{(\alpha)},|\alpha| \leq r$, and $S \in \mathcal{S e c}(M, E)$ generate the $C^{\infty}\left(T_{A} M\right)$-module $\mathcal{S e c}\left(T_{A} M, T_{A} E\right)$.

3.4. Prolongation of vector fields. Let $T_{A}$ be a Weil functor and $\kappa$ : $T_{A} \circ T \rightarrow T \circ T_{A}$ the canonical flow-natural equivalence. If $X$ is a vector field on $M$, one defines $\left(\begin{array}{c}p+r \\ r\end{array}\right)$ vector fields on $T_{A} M$ by

$$
X^{\mathrm{c}}=\kappa_{M} \circ T_{A} X, \quad X^{(\alpha)}=\kappa_{M} \circ\left(\chi_{\alpha}\right)_{T M} \circ T_{A} X \quad \text { for } 1 \leq|\alpha| \leq r .
$$

Proposition 3. Let $T_{A}$ be a Weil functor and $M$ a manifold.

(i) The vector fields $X^{(\alpha)}$ for $|\alpha| \leq r$ and $X \in \mathfrak{X}(M)$ generate $\mathfrak{X}\left(T_{A} M\right)$ over $C^{\infty}\left(T_{A} M\right)$. 
(ii) For any $X, Y \in \mathfrak{X}(M)$, we have

$$
\left[X^{(\alpha)}, Y^{(\beta)}\right]= \begin{cases}{[X, Y]^{(\alpha+\beta)}} & \text { if } 0 \leq|\alpha+\beta| \leq r \\ 0 & \text { if }|\alpha+\beta|>r .\end{cases}
$$

Proof. This is a modification of some result of [2].

3.5. Natural transformations $\bar{\chi}_{\alpha}: T_{A} \circ \otimes_{s}^{1} \rightarrow \otimes_{s}^{1} \circ T_{A}$. They are defined as follows:

$$
\left(\bar{\chi}_{\alpha}\right)_{E}\left(j_{A} \varphi\right)\left(j_{A} \eta_{1}, \ldots, j_{A} \eta_{s}\right)=\left(\chi_{\alpha}\right)_{E}\left(j_{A}\left(\varphi *\left(\eta_{1}, \ldots, \eta_{s}\right)\right)\right)
$$

for any vector bundle $E$, where $\varphi: \mathbb{R}^{p} \rightarrow \otimes_{s}^{1} E, \eta_{1}, \ldots, \eta_{s}: \mathbb{R}^{p} \rightarrow E$ are $C^{\infty}$ and

$$
\varphi *\left(\eta_{1}, \ldots, \eta_{s}\right): \mathbb{R}^{p} \rightarrow E, \quad z \mapsto \varphi(z)\left(\eta_{1}(z), \ldots, \eta_{s}(z)\right)
$$

3.6. Prolongation of tensor fields of type $(1, s)$. Let $\varphi$ be a tensor field of type $(1, s)$ on $E$. We put

$$
\varphi^{(\alpha)}=\left(\bar{\chi}_{\alpha}\right)_{E} \circ T_{A} \varphi, \quad 0 \leq|\alpha| \leq r
$$

then $\varphi^{(\alpha)}$ is a tensor field of type $(1, s)$ on $T_{A} E$. In particular if $E=T M$, we put

$$
\bar{\varphi}^{(\alpha)}=\left(\otimes_{s}^{1} \kappa_{M}\right) \circ\left(\bar{\chi}_{\alpha}\right)_{T M} \circ T_{A} \varphi, \quad 0 \leq|\alpha| \leq r
$$

then $\bar{\varphi}^{(\alpha)}$ is a tensor field of type $(1, s)$ on $T_{A} M$.

Proposition 4. Let $\varphi$ be a tensor field on $E$ of type $(1, s)$; then $\varphi^{(\alpha)}$, $0 \leq|\alpha| \leq r$, is the unique tensor field on $\left(T_{A} E, T_{A} M, T_{A} \pi\right)$ of type $(1, s)$ such that

$$
\varphi^{(\alpha)}\left(S_{1}^{\left(\alpha_{1}\right)}, \ldots, S_{s}^{\left(\alpha_{s}\right)}\right)=\left(\varphi\left(S_{1}, \ldots, S_{s}\right)\right)^{\left(\alpha+\alpha_{1}+\ldots+\alpha_{s}\right)}
$$

for any $S_{1}, \ldots, S_{s} \in \mathcal{S e c}(M, E)$ and $\alpha_{1}, \ldots, \alpha_{s} \in \mathbb{N}^{p}$ with $0 \leq\left|\alpha_{1}\right|, \ldots,\left|\alpha_{s}\right| \leq r$.

Proof. $\varphi^{(\alpha)}$ is unique by Proposition 2; moreover

$$
\begin{array}{rlr}
\varphi^{(\alpha)}( & \left.S_{1}^{\left(\alpha_{1}\right)}, \ldots, S_{s}^{\left(\alpha_{s}\right)}\right)\left(j_{A} \eta\right) & \\
& =\varphi^{(\alpha)}\left(j_{A} \eta\right)\left(S_{1}^{\left(\alpha_{1}\right)}\left(j_{A} \eta\right), \ldots, S_{s}^{\left(\alpha_{s}\right)}\left(j_{A} \eta\right)\right) & \\
& =\left(\bar{\chi}_{\alpha}\right)_{E}\left(j_{A}(\varphi \circ \eta)\right)\left(S_{1}^{\left(\alpha_{1}\right)}\left(j_{A} \eta\right), \ldots, S_{s}^{\left(\alpha_{s}\right)}\left(j_{A} \eta\right)\right) & \text { by }(7) \\
& =\left(\bar{\chi}_{\alpha}\right)_{E}\left(j_{A}(\varphi \circ \eta)\right)\left(j_{A}\left(z^{\alpha_{1}} S_{1} \circ \eta\right), \ldots, j_{A}\left(z^{\alpha_{s}} S_{s} \circ \eta\right)\right) & \text { by }(2),(3) \\
& =\left(\chi_{\alpha}\right)_{E}\left(j_{A}\left((\varphi \circ \eta) *\left(z^{\alpha_{1}} S_{1} \circ \eta, \ldots, z^{\alpha_{s}} S_{s} \circ \eta\right)\right)\right) & \text { by }(6) \\
& =\left(\chi_{\alpha}\right)_{E}\left(j_{A}\left(z^{\alpha_{1}+\ldots+\alpha_{s}}(\varphi \circ \eta) *\left(S_{1} \circ \eta, \ldots, S_{s} \circ \eta\right)\right)\right) &
\end{array}
$$




$$
\begin{array}{llr}
=j_{A}\left(z^{\alpha+\alpha_{1}+\ldots+\alpha_{s}}(\varphi \circ \eta) *\left(S_{1} \circ \eta, \ldots, S_{s} \circ \eta\right)\right) & \text { by }(2) \\
=j_{A}\left(z^{\alpha+\alpha_{1}+\ldots+\alpha_{s}} \varphi\left(S_{1}, \ldots, S_{s}\right) \circ \eta\right) & \\
=\left(\chi_{\alpha+\alpha_{1}+\ldots+\alpha_{s}}\right)_{E}\left(j_{A}\left(\varphi\left(S_{1}, \ldots, S_{s}\right) \circ \eta\right)\right) & \text { by }(2) \\
=\left(\chi_{\alpha+\alpha_{1}+\ldots+\alpha_{s}}\right)_{E}\left(T_{A} \varphi\left(S_{1}, \ldots, S_{s}\right)\left(j_{A} \eta\right)\right) & \\
=\left(\varphi\left(S_{1}, \ldots, S_{s}\right)\right)^{\left(\alpha+\alpha_{1}+\ldots+\alpha_{s}\right)}\left(j_{A} \eta\right) . &
\end{array}
$$

COROLlary 1. Let $\varphi$ be a tensor field on $M$ of type $(1, s)$; then $\bar{\varphi}^{(\alpha)}, 0 \leq$ $|\alpha| \leq r$, is the unique tensor field on $T_{A} M$ of type $(1, s)$ such that

$$
\bar{\varphi}^{(\alpha)}\left(X_{1}^{\left(\alpha_{1}\right)}, \ldots, X_{s}^{\left(\alpha_{s}\right)}\right)=\left(\varphi\left(X_{1}, \ldots, X_{s}\right)\right)^{\left(\alpha+\alpha_{1}+\ldots \alpha_{s}\right)}
$$

for any $X_{1}, \ldots, X_{s} \in \mathfrak{X}(M)$ and $\alpha_{1}, \ldots, \alpha_{s} \in \mathbb{N}^{p}$ satisfying $0 \leq\left|\alpha_{1}\right|, \ldots,\left|\alpha_{s}\right| \leq r$.

3.7. Prolongations of sections of the vector bundle $\bigwedge^{s} T^{*} M \otimes$ $\left(\otimes_{1}^{1} E\right)$ over $M$

3.7.1. Canonical morphisms $\chi_{\alpha, E}: T_{A}\left(\bigwedge^{s} T^{*} M \otimes\left(\otimes_{1}^{1} E\right)\right) \rightarrow \bigwedge^{s} T^{*} T_{A} M$ $\otimes\left(\otimes_{1}^{1} T_{A} E\right)$. Let $(E, M, \pi)$ be a vector bundle. One defines some vector bundle morphisms over $T_{A} M$,

$$
\chi_{\alpha, E}: T_{A}\left(\bigwedge^{s} T^{*} M \otimes\left(\otimes_{1}^{1} E\right)\right) \rightarrow \bigwedge^{s} T^{*} T_{A} M \otimes\left(\otimes_{1}^{1} T_{A} E\right), \quad 0 \leq|\alpha| \leq r,
$$

with natural transformations

$$
\bar{\chi}_{\alpha}: T_{A} \circ \otimes_{1}^{1} \rightarrow \otimes_{1}^{1} \circ T_{A}, \quad 0 \leq|\alpha| \leq r,
$$

by

(11) $\chi_{\alpha, E}\left(j_{A} \Phi\right)\left(\kappa_{M}\left(j_{A} \varphi_{1}\right), \ldots, \kappa_{M}\left(j_{A} \varphi_{s}\right)\right)=\left(\bar{\chi}_{\alpha}\right)_{E}\left(j_{A}\left(\Phi *\left(\varphi_{1}, \ldots, \varphi_{s}\right)\right)\right)$, where $\Phi: \mathbb{R}^{p} \rightarrow \bigwedge^{s} T^{*} M \otimes\left(\otimes_{1}^{1} E\right), \varphi_{i}: \mathbb{R}^{p} \rightarrow T M, 1 \leq i \leq s$, are $C^{\infty}$ and

$$
\Phi *\left(\varphi_{1}, \ldots, \varphi_{s}\right): \mathbb{R}^{p} \rightarrow \otimes_{1}^{1} E, \quad z \mapsto \Phi(u)\left(\varphi_{1}(z), \ldots, \varphi_{s}(z)\right) .
$$

3.7.2. Prolongation of sections. Let $(E, M, \pi)$ be a vector bundle and $R$ an $E^{*} \otimes E$-valued differential form on $M$ of degree $s$. One defines a $\left(T_{A} E\right)^{*} \otimes T_{A} E$-valued differential form $R^{(\alpha)}$ on $T_{A} M$ of degree $s$ by

$$
R^{(\alpha)}=\chi_{\alpha, E} \circ T_{A} R, \quad 0 \leq|\alpha| \leq r .
$$

REMARK 1. Assume that $E=T M$. We put

$$
\begin{aligned}
\bar{R}^{(\alpha)} & =\bigwedge^{s} \operatorname{id}_{T^{*} T_{A} M} \otimes\left(\otimes_{1}^{1} \kappa_{M}\right) \circ \chi_{\alpha, T M} \circ T_{A} R \\
& =\bigwedge^{s} \operatorname{id}_{T^{*} T_{A} M} \otimes\left(\otimes_{1}^{1} \kappa_{M}\right) \circ R^{(\alpha)} .
\end{aligned}
$$

This is a $T^{*} T_{A} M \otimes T T_{A} M$-valued differential form on $T_{A} M$ of degree $s$. We denote $\bar{R}^{(0)}$ by $R^{\mathrm{c}}$ and call it the canonical lift (or complete lift) of $R$ to $T_{A} M$. 
Proposition 5. $R^{(\alpha)}$ is the unique $\left(T_{A} E\right)^{*} \otimes T_{A} E$-valued differential form on $T_{A} M$ of degree $s$ such that

$$
R^{(\alpha)}\left(X_{1}^{\left(\beta_{1}\right)}, \ldots, X_{s}^{\left(\beta_{s}\right)}\right) S^{(\gamma)}=\left(R\left(X_{1}, \ldots, X_{s}\right) S\right)^{\left(\alpha+\beta_{1}+\ldots+\beta_{s}+\gamma\right)}
$$

for any $X_{1}, \ldots, X_{s} \in \mathfrak{X}(M), S \in \mathcal{S} e c(M, E)$ and any multi-indices $\beta_{1}, \ldots, \beta_{s}$, $\gamma \in \mathbb{N}^{p}$ satisfying $0 \leq\left|\beta_{1}\right|, \ldots,\left|\beta_{s}\right|,|\gamma| \leq r$.

Proof. We just deal with the case $s=2$. Put

$$
K=\chi_{\alpha, E}\left(j_{A}(R \circ \eta)\right)\left(\kappa_{M}\left(j_{A}\left(u^{\beta_{1}} X_{1} \circ \eta\right)\right), \kappa_{M}\left(j_{A}\left(u^{\beta_{2}} X_{2} \circ \eta\right)\right)\right) S^{(\gamma)}\left(j_{A} \eta\right) ;
$$
then

$$
\begin{aligned}
& \left(R^{(\alpha)}\left(X_{1}^{\left(\beta_{1}\right)}, X_{2}^{\left(\beta_{2}\right)}\right) S^{(\gamma)}\right)\left(j_{A} \eta\right) \\
& =R^{(\alpha)}\left(j_{A} \eta\right)\left(X_{1}^{\left(\beta_{1}\right)}\left(j_{A} \eta\right), X_{2}^{\left(\beta_{2}\right)}\left(j_{A} \eta\right)\right) S^{(\gamma)}\left(j_{A} \eta\right) \\
& =K \\
& =\left(\bar{\chi}_{\alpha}\right)_{E}\left(j_{A}\left(R \circ \eta *\left(z^{\beta_{1}} X_{1} \circ \eta, z^{\beta_{2}} X_{2} \circ \eta\right)\right)\right)\left(j_{A}\left(z^{\gamma} S \circ \eta\right)\right) \quad \text { by (11) } \\
& =\left(\chi_{\alpha}\right)_{E}\left(j_{A}\left(\left(R \circ \eta *\left(z^{\beta_{1}} X_{1} \circ \eta, z^{\beta_{2}} X_{2} \circ \eta\right)\right) * z^{\gamma} S \circ \eta\right)\right) \quad \text { by }(6) \\
& =\left(\chi_{\alpha}\right)_{E}\left(j_{A}\left(z^{\beta_{1}+\beta_{2}+\gamma} R\left(X_{1}, X_{2}\right) S \circ \eta\right)\right) \\
& =\left(\chi_{\alpha}\right)_{E}\left(\left(\chi_{\beta_{1}+\beta_{2}+\gamma}\right)_{E}\left(j_{A}\left(R\left(X_{1}, X_{2}\right) S \circ \eta\right)\right)\right) \\
& =\left(\chi_{\alpha+\beta_{1}+\beta_{2}+\gamma}\right)_{E} \circ T_{A}\left(R\left(X_{1}, X_{2}\right) S\right)\left(j_{A} \eta\right) \\
& =\left(R\left(X_{1}, X_{2}\right) S\right)^{\left(\alpha+\beta_{1}+\beta_{2}+\gamma\right)}
\end{aligned}
$$

for any $j_{A} \eta \in T_{A} M$. The uniqueness of $R^{(\alpha)}$ follows from Proposition 3 .

Corollary 2. Let $R$ be an s-differential form on $T_{A} M$ with values in $T^{*} M \otimes T M$. Then $\bar{R}^{(\alpha)}$ is the unique $T^{*} T_{A} M \otimes T T_{A} M$-differential form on $T_{A} M$ of degree $s$ such that

$$
\bar{R}^{(\alpha)}\left(X_{1}^{\left(\beta_{1}\right)}, \ldots, X_{s}^{\left(\beta_{s}\right)}\right) Y^{(\gamma)}=\left(R\left(X_{1}, \ldots, X_{s}\right) Y\right)^{\left(\alpha+\beta_{1}+\ldots \beta_{s}+\gamma\right)}
$$

for any $X_{1}, \ldots, X_{s}, Y \in \mathfrak{X}(M)$ and any multi-indices $\beta_{1}, \ldots \beta_{s}, \gamma \in \mathbb{N}^{p}$ satisfying $0 \leq\left|\beta_{1}\right|, \ldots,\left|\beta_{s}\right|,|\gamma| \leq r$.

4. Main results. We denote by $\Phi$ the vertical projection and by $K$ the connector of a linear connection $\Gamma$ on a vector bundle $(E, M, \pi)$.

Proposition 6. Let $\Gamma$ be a linear connection on a vector bundle $(E, M, \pi), \nabla$ the covariant derivative associated to $\Gamma$ and $\widetilde{\nabla}$ the covariant derivative associated to $\mathcal{T}_{A} \Gamma$. Then $\mathcal{T}_{A} \Gamma$ is the unique linear connection on $\left(T_{A} E, T_{A} M, T_{A} \pi\right)$ such that

$$
\widetilde{\nabla}_{X^{(\alpha)}} S^{(\beta)}= \begin{cases}\left(\nabla_{X} S\right)^{(\alpha+\beta)}, & \alpha, \beta \in \mathbb{N}^{p}, 0 \leq|\alpha+\beta| \leq r, \\ 0, & \alpha, \beta \in \mathbb{N}^{p},|\alpha+\beta|>r,\end{cases}
$$

where $S \in \mathcal{S e c}(M, E)$ and $X \in \mathfrak{X}(M)$. 
Proof. $\mathcal{T}_{A} \Gamma$ is unique by Propositions 2 and $3(\mathrm{i})$; moreover

$$
\begin{array}{rlr}
\tilde{\nabla}_{X^{(\alpha)}} S^{(\beta)} & =T_{A} K \circ \kappa_{E}^{-1} \circ T\left(S^{(\beta)}\right) \circ X^{(\alpha)} \\
& =T_{A} K \circ \kappa_{E}^{-1} \circ T\left(S^{(\beta)}\right) \circ \kappa_{M} \circ\left(\chi_{\alpha}\right)_{T M} \circ T_{A} X & \text { by (5) } \\
& =T_{A} K \circ \kappa_{E}^{-1} \circ T\left(\left(\chi_{\beta}\right)_{E} \circ T_{A} S\right) \circ \kappa_{M} \circ\left(\chi_{\alpha}\right)_{T M} \circ T_{A} X \quad \text { by (3) } \\
& =T_{A} K \circ \kappa_{E}^{-1} \circ T\left(\left(\chi_{\beta}\right)_{E}\right) \circ T\left(T_{A} S\right) \circ \kappa_{M} \circ\left(\chi_{\alpha}\right)_{T M} \circ T_{A} X \\
& =T_{A} K \circ \kappa_{E}^{-1} \circ T\left(\left(\chi_{\beta}\right)_{E}\right) \circ \kappa_{E} \circ T_{A}(T S) \circ\left(\chi_{\alpha}\right)_{T M} \circ T_{A} X \\
& =T_{A} K \circ\left(\chi_{\beta}\right)_{T E} \circ T_{A}(T S) \circ\left(\chi_{\alpha}\right)_{T M} \circ T_{A} X \quad \text { by Proposition } 1 \\
& =T_{A} K \circ\left(\chi_{\beta}\right)_{T E} \circ\left(\chi_{\alpha}\right)_{T E} \circ T_{A}(T S) \circ T_{A} X \\
& =\left(\chi_{\beta}\right)_{E} \circ T_{A} K \circ\left(\chi_{\alpha}\right)_{T E} \circ T_{A}(T S) \circ T_{A} X \\
& =\left(\chi_{\beta}\right)_{E} \circ\left(\chi_{\alpha}\right)_{E} \circ T_{A} K \circ T_{A}(T S) \circ T_{A} X \\
& =\left(\chi_{\alpha+\beta}\right)_{E} \circ T_{A}\left(\nabla_{X} S\right) .
\end{array}
$$

Proposition 7. Let $R_{\nabla}$ be the curvature tensor of a linear connection $\Gamma$ on $(E, M, \pi)$ and $\widetilde{\nabla}$ the covariant derivative associated to $\mathcal{T}_{A} \Gamma$. Then the curvature tensor $R_{\widetilde{\nabla}}$ of $\widetilde{\nabla}$ satisfies

$$
R_{\widetilde{\nabla}}=\left(R_{\nabla}\right)^{(0)}
$$

Proof. Since $R_{\widetilde{\nabla}}(\bar{X}, \bar{Y}) \bar{S}=\widetilde{\nabla}_{\bar{X}} \widetilde{\nabla}_{\bar{Y}} \bar{S}-\widetilde{\nabla}_{\bar{Y}} \widetilde{\nabla}_{\bar{X}} \bar{S}-\widetilde{\nabla}_{[\bar{X}, \bar{Y}]} \bar{S}$ for any $\bar{X}, \bar{Y} \in$ $\mathfrak{X}\left(T_{A} M\right)$ and $\bar{S} \in \mathcal{S e c}\left(T_{A} M, T_{A} E\right)$, we apply Propositions 3 (ii) and 6 to show that $R_{\widetilde{\nabla}}$ satisfies the conclusion of Proposition 5 .

REMARK. In particular, let $\Gamma$ be a linear connection on $M$ (i.e. $E=$ $T M)$; one can define a linear connection $\Gamma^{\mathrm{c}}$ on $T_{A} M$ by

$$
\Gamma^{\mathrm{c}}=T \kappa_{M} \circ \mathcal{T}_{A} \Gamma \circ\left(\operatorname{id}_{T T_{A} M} \times_{T_{A} M} \kappa_{M}^{-1}\right),
$$

which is called the canonical lift (or complete lift) of $\Gamma$ to $T_{A} M$. The restriction of $\Gamma^{\mathrm{c}}$ to $P^{1} M \subset T_{m}^{1} M$ (the frame bundle of $\left.M\right), m=\operatorname{dim} M$, was studied in [1].

Corollary 3. Let $\Gamma$ be a linear connection on $M, \nabla$ the covariant derivative associated to $\Gamma, \Gamma^{\mathrm{c}}$ the canonical lift of $\Gamma$ to $T_{A} M$, and $\nabla^{\mathrm{c}}$ the covariant derivative associated to $\Gamma^{\mathrm{c}}$. Then $\Gamma^{\mathrm{c}}$ is the unique linear connection on $T_{A} M$ satisfying the identities

$$
\nabla_{X^{(\alpha)}}^{\mathrm{c}} Y^{(\beta)}= \begin{cases}\left(\nabla_{X} Y\right)^{(\alpha+\beta)}, & \alpha, \beta \in \mathbb{N}^{p}, 0 \leq|\alpha+\beta| \leq r \\ 0, & \alpha, \beta \in \mathbb{N}^{p},|\alpha+\beta|>r\end{cases}
$$

where $X, Y \in \mathfrak{X}(M)$. 
COROLlary 4. Let $T_{\nabla}$ and $R_{\nabla}$ be the torsion and curvature tensors, respectively, of a linear connection $\Gamma$ on $M$, and let $\nabla^{\mathrm{c}}$ be the covariant derivative of $\Gamma^{\mathrm{c}}$. Then the torsion $T_{\nabla^{\mathrm{c}}}$ and curvature $R_{\nabla^{\mathrm{c}}}$ tensors of $\nabla^{\mathrm{c}}$ are the canonical lifts of $T_{\nabla}$ and $R_{\nabla}$ respectively, that is,

$$
T_{\nabla^{\mathrm{c}}}=\left(T_{\nabla}\right)^{\mathrm{c}}, \quad R_{\nabla^{\mathrm{c}}}=\left(R_{\nabla}\right)^{\mathrm{c}} .
$$

\section{References}

[1] L. A. Cordero, C. T. J. Dodson and M. de León, Differential Geometry of Frame Bundles, Kluwer, 1989.

[2] J. Gancarzewicz, W. M. Mikulski and Z. Pogoda, Lifts of some tensor fields and connections to product preserving functors, Nagoya Math. J. 135 (1994), 1-14.

[3] I. Kolář, P. W. Michor and J. Slovák, Natural Operations in Differential Geometry, Springer, 1993.

[4] W. M. Mikulski, Product preserving gauge bundle functors on vector bundles, Colloq. Math. 90 (2001), 277-285.

[5] K. P. Mok, Complete lifts of tensor fields and connections to the frame bundle, Proc. London Math. Soc. (3) 38 (1979), 72-88.

[6] D. J. Saunders, The Geometry of Jet Bundles, Cambridge Univ. Press, 1989.

[7] J. Slovák, Prolongations of connections and sprays with respect to Weil functors, Rend. Circ. Mat. Palermo Suppl. 14 (1987), 143-155.

École Normale Supérieure

Faculté des Sciences

Université de Yaoundé 1

Université de Yaoundé 1

B.P. 47

B.P. 812

Yaoundé, Cameroun

E-mail: achillentyam@yahoo.fr

Yaoundé, Cameroun

E-mail: wouafo@uycdc.uninet.cm

Reçu par la Rédaction le 14.10.2002

Révisé le 5.2.2003 et le 24.3.2003 